\title{
Mobile Arbeit und Ortsflexibilität: Wo arbeite ich heute, wo arbeite ich morgen?
}

\author{
Hiltraud Grzech-Sukalo \\ Online publiziert: 29. Juli 2020 \\ ๑) Springer-Verlag GmbH Deutschland, ein Teil von Springer Nature 2020
}

Liebe Leserinnen und Leser,

mobile Arbeit ist in Zeiten der Corona-Krise in aller Munde. Dies betrifft vorrangig das Arbeiten im Home-Office. Auch schon vor der Corona-Pandemie haben Unternehmen immer mehr auf diese mobile Arbeitsform gesetzt, da sie von vielen Beschäftigten als Bereicherung gesehen wird, denn das Arbeiten im Home-Office kann mit wachsender Selbstbestimmtheit einhergehen und sich positiv auf die WorkLife-Balance auswirken.

Durch die Nutzungsmöglichkeiten portabler Endgeräte gibt es neben dem Home-Office allerdings noch weitere orts- und zeitflexible und damit mobile Formen von Arbeit. Diese verschiedenen Arbeitsplatz- und Arbeitszeitmöglichkeiten führen zu positiven wie auch negativen Auswirkungen auf Gesundheit, Freizeit und Familie. So kann ein ständiges Unterwegssein, ggf. noch mit einem offenen Arbeitsende, gerade für Beschäftigte mit Sorgeverpflichtungen ein erhebliches Problem darstellen. Dies betrifft nach wie vor besonders Frauen.

Unabhängig davon sind Mobilität und Flexibilität Kernthemen der heutigen Arbeitswelt. Arbeit unter diesen Megatrends gesundheits- und sozialverträglich, aber auch attraktiv für die Beschäftigten zu gestalten, gehört zu den aktuellen Herausforderungen vieler Unternehmen. Neben den Beschäftigten selbst sind besonders Führungskräfte im Fokus, denn Führen auf Distanz bedarf besonderer Sichtweisen und Strategien.

Die im Zusammenhang mit beruflicher Mobilität bestehenden Chancen, aber auch Risiken für Beschäftigte wurden im Praxisprojekt ,prentimo - präventionsorientierte Gestaltung mobiler Arbeit" untersucht. In dem vom Bundes-

Dipl.-Psych. Hiltraud Grzech-Sukalo

hiltraud.grzech-sukalo@uni-oldenburg.de

1 Department für Wirtschafts- und Rechtswissenschaften, Carl von Ossietzky Universität Oldenburg, Ammerländer Heerstraße 114-118, 26129 Oldenburg, Deutschland ministerium für Bildung und Forschung (BMBF) geförderten Projekt arbeiteten verschiedene Forschungseinrichtungen als auch ver.di mit Unternehmen zusammen, um einerseits durch die Entwicklung von betrieblichen Gestaltungsinstrumenten die positiven Aspekte mobiler Arbeit zu sichern und andererseits Belastungen sowie Gesundheitsgefährdungen zu minimieren. Die Projektergebnisse flossen in eine Broschüre ein, die Hilfestellungen und Anregungen für eine gesundheitsorientierte Gestaltung mobiler Arbeit für betriebliche Praktiker*innen und mobil Beschäftige gibt (www.prentimo.de).

In dem hier vorliegenden Themenheft liegt nun der Fokus vorrangig auf Forschungsergebnissen zu Auswirkungen von Ortsflexibilität mobiler Arbeit. Diese stammen in erster Linie, aber nicht nur, aus dem Projekt prentimo. Bei den betrachteten Formen von Mobilität handelt es sich um Arbeit im Home-Office, aber auch um Dienstreisen, Außendienst oder Arbeiten in „,modernen“ Bürowelten.

Der erste Beitrag von Sylvia Kraus, Hiltraud Grzech-Sukalo und Kerstin Rieder geht der Frage nach, welche Formen ortsflexibler Arbeit besonders mit Belastungen für die Beschäftigten verbunden sind. Dies wurde im Rahmen des Projektes prentimo in einem Unternehmen im Bereich Facility-Management untersucht. Die Ergebnisse zeigen, dass mobile Arbeit in Form von täglich mehrfach wechselnden Einsatzorten mit signifikant höheren Belastungen, geringeren Ressourcen, höherer interessierter Selbstgefährdung und verstärkten Konflikten zwischen Erwerbsarbeit und Privatleben einhergeht. Gerade Kombinationen mit verschiedenen berufsbedingten Mobilitätsformen zeigen deutliche negative Auswirkungen, besonders in der Kombination mit ungünstig gestalteten Arbeitszeiten.

Anna Monz und Gerlinde Vogl widmen sich dem Themenfeld ortsgebundener mobiler Arbeit von Techniker*innen, die die Anwesenheit bei Kund*innen zwingend voraussetzt. Anhand der Auswertung detaillierter Interviews, die im Rahmen des Projektes prentimo erhoben wurden, werden neue Anforderungen analysiert, die im Zusammenhang mit der Digitalisierung stehen und sich sowohl auf 
die Tätigkeit und den Arbeitsprozess selbst als auch auf die räumliche und digitale Mobilität auswirken.

Thomas Breisig zeigt in seinem Praxisbeitrag zu mobiler Arbeit und Führung einen Überblick, welche Faktoren und Rahmenbedingungen Führung auf Distanz prägen. Darüber hinaus beschäftigt er sich mit Voraussetzungen und Haltungen, die den Akteur*innen abverlangt werden und thematisiert, wie Unternehmen Führungskräfte als auch Mitarbeiter*innen bei einer erfolgreichen Umsetzung unterstützen können. Die Aussagen werden durch empirische Befunde aus dem Forschungsprojekt prentimo belegt.

Der Beitrag von Tanja Carstensen stammt aus dem Forschungsprojekt „Wandel der Geschlechterverhältnisse durch Digitalisierung“ der Hans-Böckler-Stiftung. Untersuchungsschwerpunkt ist, ob und wie sich bei ortsund zeitflexiblem Arbeiten mit digitalen Technologien Vereinbarkeit von Beruf und Familie und Geschlechterungleichheiten in der häuslichen Arbeitsteilung verändern. Es werden Hinweise gegeben auf förderliche und hinderliche Bedingungen für einen Abbau der Ungleichheiten in der Zuständigkeit und der Arbeitsteilung im Beruf und der Familie.

Der Artikel von Sarah S. Lütke Lanfer und Cathrin Becker präsentiert zentrale Ergebnisse, die im Rahmen des Projektes „PRÄGEWELT - Präventionsorientierte Gestaltung neuer Open Space-Arbeitswelten“ (gefördert vom BMBF; www.praegwelt.de) gewonnen wurden. Digitali- sierung, Flexibilisierung und Kostendruck führten in den vergangenen Jahren zunehmend dazu, dass Unternehmen Büroräume in offene Büroflächen mit unterschiedlichen Arbeitsbereichen oder informellen Aufenthaltsmöglichkeiten umgestalteten. Derzeitige Studien belegen, dass gerade eine ungünstig gestaltete physische Arbeitsumgebung eine der häufigsten Ursachen für Fehlzeiten durch psychische Erkrankungen ist. Diese Studien werden nach verschiedenen Kategorien, wie Arbeitsaufgabe, Organisation und Person in Beziehung gesetzt und analysiert.

In den Artikeln zeigt sich fast durchgehend, dass hinsichtlich mobiler Arbeit nach wie vor Forschungsbedarf besteht. Hierzu sollten zukünftig neben den Anforderungen, die sich aus der konkreten Arbeitstätigkeit ergeben, auch immer die mobilitätsbedingten Aspekte gleichermaßen mit analysiert werden. Dazu gehören neben den verschiedenen Orten, an denen gearbeitet wird, auch Geschlechterarrangements in Betreuungs- und Sorgearbeit. Zudem ist das Führungsverhalten der Vorgesetzten in Unternehmen zu berücksichtigen und zu analysieren. Gleichzeitig gilt es, die zeitlichen Aspekte wie Dauer, Lage und Verteilung der realen Arbeitszeiten mobiler Arbeit einzubeziehen. Erst durch eine systematische Analyse des Zusammenwirkens dieser verschiedenen Aspekte ergeben sich spezifische Erkenntnisse, aus denen fundierte Empfehlungen zur Minimierung bestehender Belastungen bei mobiler Arbeit abgeleitet werden können. 